\title{
Dynamics of a Stage Structured Pest Control Model in a Polluted Environment with Pulse Pollution Input
}

\author{
Bing Liu, ${ }^{1}$ Ling $\mathrm{Xu},{ }^{2}$ and Baolin Kang ${ }^{1}$ \\ ${ }^{1}$ Department of Mathematics, Anshan Normal University, Anshan, Liaoning 114007, China \\ ${ }^{2}$ Department of Mathematics, Liaoning Normal University, Dalian, Liaoning 116029, China \\ Correspondence should be addressed to Bing Liu; liubing529@126.com
}

Received 18 December 2012; Revised 3 August 2013; Accepted 23 August 2013

Academic Editor: Maoan Han

Copyright (c) 2013 Bing Liu et al. This is an open access article distributed under the Creative Commons Attribution License, which permits unrestricted use, distribution, and reproduction in any medium, provided the original work is properly cited.

\begin{abstract}
By using pollution model and impulsive delay differential equation, we formulate a pest control model with stage structure for natural enemy in a polluted environment by introducing a constant periodic pollutant input and killing pest at different fixed moments and investigate the dynamics of such a system. We assume only that the natural enemies are affected by pollution, and we choose the method to kill the pest without harming natural enemies. Sufficient conditions for global attractivity of the natural enemy-extinction periodic solution and permanence of the system are obtained. Numerical simulations are presented to confirm our theoretical results.
\end{abstract}

\section{Introduction}

Nowadays, the problem of the world's environmental pollution is serious, which has a frustrating effect on the ecosystem damage in the direct or indirect ways. Pollution leads to the living environmental change and gene mutation. It results in not only birth defects and deformities but also population variability, which decreases the number of the population in the nature and even makes them extinct. In order to assess the risk of the populations exposed to a polluted environment, in recent years, mathematical models concerning this topic have been studied extensively including continuous pollution input and impulsive pollution input [1-11].

As we all know, the predator-prey system can be used to model the process of controlling the pests by spraying pesticides, as well as relying on their natural enemies. However, in a polluted environment, some natural enemies are affected by pollution seriously and pests almost are not affected. For example, frogs are the natural enemies of beetles, locusts, and mole cricket, but some chemical plants discard waste products into rivers for their convenience, which cause severe water contamination, seriously injures frog's reproductive system, and significantly decreases their fertility. Moreover, water pollution also causes large quantities of the fertilized eggs and tadpoles to die, resulting in the decrease of frogs. It is shown in a Sweden's new study that male tadpoles can eventually grow into female frogs only in the environment similar to the nature but full of pollutants with estrogen. However, some male frogs have ovaries but no fallopian tubes, and they finally turn into lifelong infertile frogs, which are called "Yin and Yang frog", and nearly one-third of the world's frog species may be extinct because of the environmental pollution. People must control the period and quantity of emission of pollution to prevent natural enemy from extinction. In addition, too much pesticide spraying will reduce pests significantly; meanwhile, it also causes serious environmental pollution. Therefore, when controlling pests, we had better choose the method to kill the pests without polluting the environment and harming natural enemies at regular intervals.

The predator-prey models with stage structure for the predator were introduced or investigated by Hastings and Wang [12-14]. Since the immature predator takes $\tau$ (which is called maturation time delay) units of time to mature, the death toll during the juvenile period should be considered, and time delays have important biological meanings in stage structured models. Recently, many models with time delay were extensively studied [15-22]. 
According to the above biological background, in this paper, we suggest an impulsive predator-prey pollution model with stage structured for predator by introducing a constant periodic pollutant input and proportional killing pest at different fixed moments to model the process of pest control and polluted environment. Recently, there has been quite a lot of literatures on the applications of impulsive differential equations on population $[1,2,8,10,11,20-31]$. To our knowledge, there have been no results on this topic in the literature. The questions that arise here are as follows: how do we control the emission of pollution to prevent the extinction of natural enemies? Under what condition can the system be permanent? How can we control pests effectively?

The organization of this paper is as follows. In the next section, we formulate our model and give several lemmas which are useful for our main results. In Section 3 and Section 4, the sufficient conditions for the global attractivity of the "natural enemy-extinction" periodic solution and permanence of the system are obtained. We give a brief discussion of our results in Section 5. Numerical simulations are presented to illustrate our theoretical results.

\section{Model Formulation and Preliminaries}

In this paper, we assume only that the natural enemies are affected by pollution and we choose the method to kill the pest without harming natural enemies. Then a pest control model with stage structure for natural enemy in a polluted environment by introducing a constant periodic pollutant input and killing pests at different fixed moment is formulated as follows:

$$
\begin{aligned}
& \frac{d x(t)}{d t}=\beta x(t)\left(1-\frac{x(t)}{K}\right)-\frac{q x(t) y_{2}(t)}{1+\alpha x(t)}, \\
& \frac{d y_{1}(t)}{d t}=\lambda \frac{q x(t) y_{2}(t)}{1+\alpha x(t)} \\
& -\lambda e^{-d \tau} \frac{q x(t-\tau) y_{2}(t-\tau)}{1+\alpha x(t-\tau)} \\
& -d y_{1}(t)-f_{1} c_{0}(t) y_{1}(t) \text {, } \\
& \frac{d y_{2}(t)}{d t}=\lambda e^{-d \tau} \frac{q x(t-\tau) y_{2}(t-\tau)}{1+\alpha x(t-\tau)} \\
& -\gamma y_{2}(t)-f_{2} c_{0}(t) y_{2}(t) \\
& \frac{d c_{0}(t)}{d t}=k c_{e}(t)-g c_{0}(t)-m c_{0}(t) \\
& \frac{d c_{e}(t)}{d t}=-h c_{e}(t) \\
& t \neq(n+l-1) T, t \neq n T, \\
& \Delta x(t)=0, \quad \Delta y_{1}(t)=0, \quad \Delta y_{2}(t)=0, \\
& \Delta c_{0}(t)=0, \quad \Delta c_{e}(t)=b, \\
& t=(n+l-1) T,
\end{aligned}
$$

$$
\begin{array}{rrr}
\Delta x(t)=-\delta x(t), \quad \Delta y_{1}(t)=0, & \Delta y_{2}(t)=0, \\
\Delta c_{0}(t)=0, \quad \Delta c_{e}(t)=0, & t=n T,
\end{array}
$$

where $0 \leq l \leq 1, \Delta x(t)=x\left(t^{+}\right)-x(t), \Delta y_{i}(t)=y_{i}\left(t^{+}\right)-$ $y_{i}(t)(i=1,2), \Delta c_{0}(t)=c_{0}\left(t^{+}\right)-c_{0}(t), \Delta c_{e}(t)=c_{e}\left(t^{+}\right)-$ $c_{e}(t) . x(t), y_{1}(t)$, and $y_{2}(t)$ represent the densities of prey (pest), immature, and mature predator (natural enemy) at time $t$, respectively; $c_{e}(t), c_{0}(t)$ represent the concentration of pollution in the environment and organism at time $t$, respectively; $\beta$ is intrinsic growth rate of the pests in the absence of natural enemies; $K>0$ is the pest capacity of environment; $q$ is the predation rate of natural enemy and $\lambda$ represents the conversion rate at which ingested pest in excess of what is needed for maintenance is translated into natural enemy increase; $\alpha$ is the saturation which represents that a certain amount of natural enemies can prey on a limited amount of pests, though the pests are numerous; $d$ and $\gamma$ are the death rate of immature and mature natural enemies, respectively; in addition, we assume that juveniles suffer a mortality rate of $d$ (the through-stage death rate) and take $\tau$ units of time to mature. $f_{1}$ and $f_{2}$ are the dose-response parameters of species to the pollution in the immature and mature natural enemies, respectively; the exogenous quantity of impulsive input of pollutant into the environment at time $t=(n+l-1) T$ is represented by $b ; \delta(0 \leq \delta<1)$ represents a proportional decrease of pest because of being harvested at time $t=n T$. The other parameters can be seen in [1].

The initial conditions for (2) are

$$
\begin{aligned}
\left(x(t), y_{1}(t), y_{2}(t), c_{0}(t), c_{e}(t)\right) \\
=\left(\varphi_{1}(t), \varphi_{2}(t), \varphi_{3}(t), \varphi_{4}(t), \varphi_{5}(t)\right) \in C_{+} \\
=C\left([-\tau, 0], R_{+}^{5}\right), \quad \varphi_{i}(0)>0, i=1,2,3,4,5 .
\end{aligned}
$$

Note that the variable $y_{1}(t)$ does not appear in the first, third, forth, and fifth equations of system (2); hence, we only need to consider the subsystem of (2) as follows:

$$
\begin{gathered}
\frac{d x(t)}{d t}=\beta x(t)\left(1-\frac{x(t)}{K}\right)-\frac{q x(t) y_{2}(t)}{1+\alpha x(t)}, \\
\frac{d y_{2}(t)}{d t}=\lambda e^{-d \tau} \frac{q x(t-\tau) y_{2}(t-\tau)}{1+\alpha x(t-\tau)} \\
\quad-\gamma y_{2}(t)-f_{2} c_{0}(t) y_{2}(t), \\
\frac{d c_{0}(t)}{d t}= \\
k c_{e}(t)-g c_{0}(t)-m c_{0}(t), \\
\frac{d c_{e}(t)}{d t}=-h c_{e}(t), \\
t \neq(n+l-1) T, t \neq n T,
\end{gathered}
$$




$$
\begin{array}{r}
\Delta x(t)=0, \quad \Delta y_{2}(t)=0, \\
\Delta c_{0}(t)=0, \quad \Delta c_{e}(t)=b, \\
t=(n+l-1) T \\
\Delta x(t)=-\delta x(t), \quad \Delta y_{2}(t)=0, \\
\Delta c_{0}(t)=0, \quad \Delta c_{e}(t)=0, \\
t=n T .
\end{array}
$$

Lemma 1 (see [32]). Consider the following delay differential equation:

$$
\frac{d x(t)}{d t}=r_{1} x(t-\tau)-r_{2} x(t)
$$

where $r_{1}, r_{2}$, and $\tau$ are all positive constants and $x(t)>0$ for $-\tau \leq t \leq 0$; we have
(1) if $r_{1}<r_{2}$, then $\lim _{t \rightarrow+\infty} x(t)=0$;
(2) if $r_{1}>r_{2}$, then $\lim _{t \rightarrow+\infty} x(t)=+\infty$.

Lemma 2 (see [1]). Consider the following subsystem of (2):

$$
\begin{gathered}
\frac{d c_{0}(t)}{d t}=k c_{e}(t)-g c_{0}(t)-m c_{0}(t), \\
\frac{d c_{e}(t)}{d t}=-h c_{e}(t), \\
t \neq(n+l-1) T, \\
\Delta c_{0}(t)=0, \quad \Delta c_{e}(t)=b, \quad t=(n+l-1) T .
\end{gathered}
$$

Then system (5) has a unique positive T-periodic solution $\left(c_{0}^{*}(t), c_{e}^{*}(t)\right)$, which is globally asymptotically stable, where

$$
\begin{gathered}
c_{0}^{*}(t)=c_{0}^{*}(0) e^{-(g+m)(t-(n+l-1) T)} \\
+\frac{k b\left(e^{-(g+m)(t-(n+l-1) T}-e^{-h(t-(n+l-1) T}\right)}{(h-g-m)\left(1-e^{-h T}\right)}, \\
c_{e}^{*}(t)=\frac{b e^{-h(t-(n+l-1) T)}}{1-e^{-h T}}, \\
k b\left(e^{-(g+m) T}-e^{-h T}\right) \\
c_{e}^{*}(0)=\frac{b}{1-e^{-h T}},
\end{gathered}
$$

and $t \in((n+l-1) T,(n+l) T]$.

Lemma 3. There exists a constant $L>0$ such that $x(t) \leq K$, $y_{1}(t) \leq L, y_{2}(t) \leq L, c_{0}(t) \leq L$, and $c_{e}(t) \leq L$.

Proof. Define $V(t)=\lambda x(t)+y_{1}(t)+y_{2}(t)+c_{0}(t)+c_{e}(t)$. Since $d x(t) / d t \leq \beta x(t)(1-(x / K)),\left.\dot{x}\right|_{x=K} \leq 0$, in addition, $0 \leq \delta<1, x\left(n T^{+}\right) \leq x(n T)$, thus $x(t) \leq K$ for $t$ large enough.
Define $d^{*}=\min \{d, \gamma, g+m, h-k\}$; then, for $t \neq(n+l-1) T$, $t \neq n T$; we have

$$
\frac{d V(t)}{d t}<L_{0}-d^{*} V(t)
$$

where $L_{0}=\lambda K\left(\beta+d^{*}\right)^{2} / 4 \beta$. Consider the following impulse differential inequalities:

$$
\begin{gathered}
\frac{d V(t)}{d t} \leq-d^{*} V(t)+L_{0}, \quad t \neq(n+l-1) T, t \neq n T, \\
V\left((n+l-1) T^{+}\right)=V((n+l-1) T)+b, \\
t=(n+l-1) T, \\
V\left(n T^{+}\right) \leq V(n T), \quad t=n T .
\end{gathered}
$$

We have

$$
\begin{aligned}
V(t) \leq & V\left(0^{+}\right) e^{-d^{*} t}+\int_{0}^{t} L_{0} e^{-d^{*}(t-s)} d s \\
& +\sum_{0<(n+l-1) T<l} b e^{-d^{*}(t-(n+l-1) T)} .
\end{aligned}
$$

Hence

$$
\lim _{t \rightarrow+\infty} V(t) \leq \frac{L_{0}}{d^{*}}+\frac{b e^{d^{*} l T}}{e^{d^{*} T}-1}=L,
$$

so $V(t)$ is uniformly ultimately bounded. Therefore, by the definition of $V(t)$, system (2) is uniformly ultimately bounded. The proof is completed.

Lemma 4 (see [29]). If $\delta<1-e^{-\beta T}$ holds, system

$$
\begin{gathered}
\frac{d x(t)}{d t}=\beta x(t)\left(1-\frac{x(t)}{K}\right), \quad t \neq n T, \\
x\left(t^{+}\right)=(1-\delta) x(t), \quad t=n T
\end{gathered}
$$

has a unique positive globally asymptotically stable periodic solution $x^{*}(t)=K\left(1-\delta-e^{-\beta T}\right) /\left(1-\delta-e^{-\beta T}+\delta e^{-\beta(t-n T)}\right)$, $t \in(n T,(n+1) T]$.

Therefore, if $\delta<1-e^{-\beta T}$ holds, the system (2) has a natural enemy-extinction periodic solution $\left(x^{*}(t), 0, c_{0}^{*}(t), c_{e}^{*}(t)\right)$. In this paper, we assume that $\delta<1-e^{-\beta T}$ always holds.

Remark 5 (see [1]). $c_{0}(t)$ and $c_{e}(t)$ are the concentration of pollution. To assure $0 \leq c_{0}(t) \leq 1$ and $0 \leq c_{e}(t) \leq 1$, it is necessary that $g \leq k \leq g+m, b \leq 1-e^{-h T}$.

Remark 6 (see [1]). According to the biological significance, we assume $k<h$.

\section{Global Attractivity of the "Natural Enemy-Extinction" Periodic Solution}

In this section, we discuss under what condition the natural enemies will go extinct. 
Denote

$$
R_{1}=\frac{\lambda q K e^{-d \tau}}{\left(\gamma+f_{2} \rho\right)\left(\alpha K+g_{1}(\delta, T)\right)}
$$

where

$$
\begin{gathered}
\rho=\frac{k b\left(e^{-(g+m) T}-e^{-h T}\right) e^{-(g+m) T}}{(h-g-m)\left(1-e^{-(g+m) T}\right)\left(1-e^{-h T}\right)}, \\
g_{1}(\delta, T)=\frac{(1-\delta)\left(1-e^{-\beta T}\right)}{1-\delta-e^{-\beta T}} .
\end{gathered}
$$

Theorem 7. If $R_{1}<1$, then the "mature natural enemyextinction" periodic solution $\left(x^{*}(t), 0, c_{0}^{*}(t), c_{e}^{*}(t)\right)$ of system (2) is globally attractive.

Proof. Since $R_{1}<1$, we have

$$
e^{-d \tau} \frac{\lambda q K\left(1-\delta-e^{-\beta T}\right)}{\left(1-e^{-\beta T}\right)(1-\delta)+\alpha K\left(1-\delta-e^{-\beta T}\right)}<\gamma+f_{2} \rho .
$$

By Lemma 2, for sufficiently small enough $\varepsilon_{1}>0$, there exists a positive constant $N_{1}$ such that

$$
c_{0}(t)>c_{0}^{*}(t)-\varepsilon_{1}>\rho-\varepsilon_{1}>0 .
$$

holds for $t \geq N_{1} T$.

Note that

$$
\begin{gathered}
\frac{d x(t)}{d t} \leq \beta x(t)\left(1-\frac{x(t)}{K}\right), \quad t \neq n T, n \in Z^{+}, \\
\Delta x(t)=-\delta x(t), \quad t=n T, n \in Z^{+} .
\end{gathered}
$$

Then we consider the following comparison system:

$$
\begin{gathered}
\frac{d z(t)}{d t}=\beta z(t)\left(1-\frac{z(t)}{K}\right), \quad t \neq n T, n \in Z^{+}, \\
\Delta z(t)=-\delta z(t), \quad t=n T, n \in Z^{+} .
\end{gathered}
$$

According to Lemma 4, we know that

$$
\begin{array}{r}
z^{*}(t)=x^{*}(t)=\frac{K\left(1-\delta-e^{-\beta T}\right)}{1-\delta-e^{-\beta T}+\delta e^{-\beta(t-n T)}}, \\
t \in(n T,(n+1) T]
\end{array}
$$

is a unique globally asymptotically stable positive $T$-periodic solution of system (17).

By using comparison theorem of impulsive differential equation, there exist a positive integer $N_{2}$ and a sufficiently small positive constant $\varepsilon_{2}$ such that for all $n T<t \leq(n+$ 1) $T, n>N_{2}$,

$$
\begin{aligned}
x(t) & \leq x^{*}(t)+\varepsilon_{2} \\
& \leq \frac{K\left(1-\delta-e^{-\beta T}\right)}{\left(1-e^{-\beta T}\right)(1-\delta)}+\varepsilon_{2}=\eta
\end{aligned}
$$

holds. From (15), (19), and the second equation of (2), we obtain that for $t>N T+\tau$,

$$
\begin{aligned}
\frac{d y_{2}(t)}{d t} \leq & \lambda e^{-d \tau} \frac{q \eta}{1+\alpha \eta} y_{2}(t-\tau) \\
& -\left(\gamma+f_{2}\left(\rho-\varepsilon_{1}\right)\right) y_{2}(t)
\end{aligned}
$$

holds.

Consider the following comparison equation:

$$
\begin{aligned}
\frac{d u(t)}{d t}= & \lambda e^{-d \tau} \frac{q \eta}{1+\alpha \eta} u(t-\tau) \\
& -\left(\gamma+f_{2}\left(\rho-\varepsilon_{1}\right)\right) u(t) .
\end{aligned}
$$

By inequality (14), we have that $\lambda e^{-d \tau} q \eta /(1+\alpha \eta)<\gamma+$ $f_{2}\left(\rho-\varepsilon_{1}\right)$ holds; then, according to Lemma 1 , we obtain that

$$
\lim _{t \rightarrow+\infty} u(t)=0
$$

By the comparison theorem of delay differential equation, we have $\lim _{t \rightarrow+\infty} y_{2}(t)=0$.

Without loss of generality, we may assume that $0<$ $y_{2}(t)<\varepsilon_{3}\left(\varepsilon_{3}\right.$ is sufficiently small positive constant such that $\left.\delta<1-e^{-\left(\beta-q \varepsilon_{3}\right) T}\right)$ for all $t \geq 0$; by the first equation of system (2), we have

$$
\begin{aligned}
\frac{d x(t)}{d t} & \geq \beta x(t)\left(1-\frac{x(t)}{K}\right)-\frac{q x(t) \varepsilon_{3}}{1+\alpha x(t)} \\
& \geq \beta x(t)\left(1-\frac{x(t)}{K}\right)-q x(t) \varepsilon_{3} .
\end{aligned}
$$

Consider the following comparison equation:

$$
\begin{gathered}
\frac{d z_{1}(t)}{d t}=\beta z_{1}(t)\left(1-\frac{z_{1}(t)}{K}\right)-q z_{1}(t) \varepsilon_{3} \\
=\left(\beta-q \varepsilon_{3}\right) z_{1}(t)\left(1-\frac{z_{1}(t)}{K\left(\beta-q \varepsilon_{3}\right) / \beta}\right), \\
\quad t \neq n T, n \in Z^{+}, \\
\Delta z_{1}(t)=-\delta z_{1}(t), \quad t=n T, n \in Z^{+} .
\end{gathered}
$$

By Lemma 4,

$$
\begin{array}{r}
z_{1}^{*}(t)=\frac{\left(K\left(\beta-q \varepsilon_{3}\right) / \beta\right)\left(1-\delta-e^{-\left(\beta-q \varepsilon_{3}\right) T}\right)}{1-\delta-e^{-\left(\beta-q \varepsilon_{3}\right) T}+\delta e^{-\left(\beta-q \varepsilon_{3}\right)(t-n T)}} \\
t \in(n T,(n+1) T]
\end{array}
$$

is a unique globally asymptotically stable positive $T$-periodic solution of system (24). By using comparison theorem of impulsive differential equation, for above $\varepsilon_{2}$ and $t$ large enough, we have

$$
x(t)>z_{1}^{*}(t)-\varepsilon_{2} .
$$


It follows from (19) and (26) that

$$
z_{1}^{*}(t)-\varepsilon_{2}<x(t)<x^{*}(t)+\varepsilon_{2},
$$

holds for $t$ large enough. Let $\varepsilon_{3} \rightarrow 0$; we can get $z_{1}^{*}(t) \rightarrow$ $x^{*}(t)$, so

$$
x^{*}(t)-\varepsilon_{2}<x(t)<x^{*}(t)+\varepsilon_{2}
$$

holds for $t$ large enough, which implies $x(t) \rightarrow x^{*}(t)$ as $t \rightarrow$ $+\infty$. According to Lemma 2, $c_{0}(t) \rightarrow c_{0}^{*}(t), c_{e}(t) \rightarrow c_{e}^{*}(t)$ as $t \rightarrow+\infty$. This completes the proof.

\section{Permanence}

Definition 8. System (2) is said to be permanent if there are positive constants $m, M$, and a finite time $T_{0}$ such that for all solutions $\left(x(t), y_{1}(t), y_{2}(t), c_{0}(t), c_{e}(t)\right)$ with initial conditions $(2), m \leq x(t) \leq M, m \leq y_{i}(t) \leq M, m \leq c_{0}(t) \leq M, m \leq$ $c_{e}(t) \leq M$ holds for all $t \geq T_{0}, i=1,2$.

Denote

$$
R_{2}=\frac{\lambda q K e^{-d \tau}}{\left(\gamma+f_{2} B\right)\left(\alpha K+g_{2}(\delta, T)\right)},
$$

where

$$
\begin{gathered}
B=\frac{k b\left(e^{-(g+m) T}-e^{-h T}\right)}{(h-g-m)\left(1-e^{-(g+m) T}\right)\left(1-e^{-h T}\right)} \\
+\frac{k b}{|h-g-m|\left(1-e^{-h T}\right)}, \\
g_{2}(\delta, T)=\frac{1-e^{-\beta T}}{1-\delta-e^{-\beta T}} .
\end{gathered}
$$

Theorem 9. If $R_{2}>1$, there exists a positive constant $m^{*}$ such that $y_{2}(t) \geq m^{*}$ for any solution $\left(x(t), y_{2}(t), c_{0}(t), c_{e}(t)\right)$ of system (2).

Proof. Since $R_{2}>1$, we can choose positive constants $m_{1}^{*}, \epsilon_{1}$, and $\epsilon_{2}$ such that

$$
\lambda q e^{-d \tau} \frac{\sigma}{1+\alpha \sigma}>\gamma+f_{2}\left(B+\epsilon_{2}\right)
$$

and $\delta<1-e^{-\left(\beta-q m_{1}^{*}\right) T}$ hold, where

$$
\begin{aligned}
\sigma= & \frac{K\left(\beta-q m_{2}^{*}\right)\left(1-\delta-e^{-\left(\beta-q m_{1}^{*}\right) T}\right)}{\beta\left(1-e^{-\left(\beta-q m_{2}^{*}\right) T}\right)} \\
& -\epsilon_{1}>0 .
\end{aligned}
$$

The second equation of system (2) can be written as

$$
\begin{aligned}
\frac{d y_{2}(t)}{d t}= & \left(\lambda q e^{-d \tau} \frac{x(t)}{1+\alpha x(t)}-\gamma-f_{2} c_{0}(t)\right) y_{2}(t) \\
& -\lambda q e^{-d \tau} \frac{d}{d t} \int_{t-\tau}^{t} \frac{x(\theta)}{1+\alpha x(\theta)} y_{2}(\theta) d \theta
\end{aligned}
$$

Define

$$
V(t)=y_{2}(t)+\lambda q e^{-d \tau} \frac{d}{d t} \int_{t-\tau}^{t} \frac{x(\theta)}{1+\alpha x(\theta)} y_{2}(\theta) d \theta
$$

Calculating the derivative of $V(t)$ along the solution of (2), we have

$$
\frac{d V(t)}{d t}=\left(\lambda q e^{-d \tau} \frac{x(t)}{1+\alpha x(t)}-\gamma-f_{2} c_{0}(t)\right) y_{2}(t)
$$

We claim that the inequality $y_{2}(t)<m_{1}^{*}$ cannot hold for all $t \geq t_{0}$. Otherwise, there is a positive constant $t_{0}$ such that $y_{2}(t)<m_{1}^{*}$ for all $t \geq t_{0}$. From the first equation of system (2), we have

$$
\begin{array}{r}
\frac{d x(t)}{d t}>\beta x(t)\left(1-\frac{x(t)}{K}\right)-q x(t) m_{2}^{*}, \\
t \neq n T, n \in Z^{+}, \\
\Delta x(t)=-\delta x(t), \quad t=n T, n \in Z^{+} .
\end{array}
$$

Consider the following comparison system:

$$
\begin{gathered}
\frac{d z(t)}{d t}=\beta z(t)\left(1-\frac{z(t)}{K}\right)-q z(t) m_{2}^{*}, \\
t \neq n T, n \in Z^{+}, \\
\Delta z(t)=-\delta z(t), \quad t=n T, n \in Z^{+} .
\end{gathered}
$$

Then

$$
\begin{array}{r}
z^{*}(t)=\frac{\left(K\left(\beta-q m_{2}^{*}\right) / \beta\right)\left(1-\delta-e^{-\left(\beta-q m_{2}^{*}\right) T}\right)}{1-\delta-e^{-\left(\beta-q m_{2}^{*}\right) T}+\delta e^{-\left(\beta-q m_{2}^{*}\right)(t-n T)}} \\
t \in(n T,(n+1) T]
\end{array}
$$

is a unique globally asymptotically stable positive $T$-periodic solution of system (37). By using comparison theorem of impulsive differential equation, for $\epsilon_{1}>0$, there exists a $T_{1}>t_{0}$ such that for $t>T_{1}$,

$$
\begin{aligned}
x(t) & >z^{*}(t)-\epsilon_{1} \\
& \geq \frac{K\left(\beta-q m_{2}^{*}\right)\left(1-\delta-e^{-\left(\beta-q m_{2}^{*}\right) T}\right)}{\beta\left(1-e^{-\left(\beta-q m_{2}^{*}\right) T}\right)}-\epsilon_{1} \\
& =\sigma
\end{aligned}
$$

holds.

By Lemma 2, for $\epsilon_{2}>0$, there exists a $T_{2}>0$ such that for $t>T_{2}$,

$$
c_{0}^{*}(t)-\varepsilon_{2}<c_{0}(t)<c_{0}^{*}(t)+\varepsilon_{2}<B+\varepsilon_{2} .
$$


Let $T_{0}=\max \left\{T_{1}, T_{2}\right\}$, and from (39) and (40), we have

$$
\begin{array}{r}
\frac{d V(t)}{d t}>\left(\lambda q e^{-d \tau} \frac{\sigma}{1+\alpha \sigma}-\gamma-f_{2}\left(B+\epsilon_{2}\right)\right) y_{2}(t), \\
t>T_{0} .
\end{array}
$$

Let

$$
y_{2}^{l}=\min _{t \in\left[T_{0}, T_{0}+\tau\right]}\left\{y_{2}(t)\right\}
$$

We will show that $y_{2}(t) \geq y_{2}^{l}$ for all $t \geq T_{0}$. Otherwise, there exists a nonnegative constant $T_{3}$ such that $y_{2}(t) \geq y_{2}^{l}$ for $t \epsilon$ $\left[T_{0}, T_{0}+T_{3}+\tau\right], y_{2}\left(T_{0}+T_{3}+\tau\right)=y_{2}^{l}$ and $d y_{2}\left(T_{0}+T_{3}+\tau\right) / d t<0$. Thus from the second equation of (2), (31), and (41), we easily see that

$$
\begin{aligned}
& \frac{d y_{2}\left(T_{0}+T_{3}+\tau\right)}{d t} \\
& \quad \geq y_{2}^{l}\left[\lambda q e^{-d \tau} \frac{\sigma}{1+\alpha \sigma}-\gamma-f_{2}\left(B+\epsilon_{2}\right)\right] \\
& \quad>0,
\end{aligned}
$$

which is a contradiction. Hence we get that $y_{2}^{l} \geq m_{1}^{*}$ for all $t>T_{0}$. Then we have

$$
\begin{aligned}
\frac{d V(t)}{d t} & >y_{2}^{l}\left(\lambda q e^{-d \tau} \frac{\sigma}{1+\alpha \sigma}-\gamma-f_{2}\left(B+\epsilon_{2}\right)\right) \\
& >0, \quad t>T_{0},
\end{aligned}
$$

which implies $V(t) \rightarrow+\infty$ as $t \rightarrow+\infty$. This is a contradiction to $V(t) \leq L+K L \lambda q e^{-d \tau} \tau$. Therefore, for any positive constant $t_{0}$, the inequality $y_{2}(t)<m_{1}^{*}$ cannot hold for all $t \geq t_{0}$. If $y_{2}(t) \geq m_{1}^{*}$ holds true for all $t$ large enough, then our aim is obtained; otherwise, $y_{2}(t)$ is oscillatory about $m_{1}^{*}$. Let

$$
m^{*}=\min \left\{\frac{m_{1}^{*}}{2}, m_{1}^{*} e^{-\left(\gamma+f_{2} L\right) \tau}\right\} .
$$

In the following, we will show that $y_{2}(t) \geq m^{*}$. There exist two positive constants $t^{*}$ and $\theta^{*}$ such that

$$
\begin{gathered}
y_{2}\left(t^{*}\right)=y_{2}\left(t^{*}+\theta^{*}\right)=m_{1}^{*}, \\
y_{2}(t)<m_{1}^{*}, \quad t^{*}<t<t^{*}+\theta^{*} .
\end{gathered}
$$

When $t^{*}$ is large enough, the inequality $x(t)>\sigma$ holds true for $t^{*}<t<t^{*}+\theta^{*}$. Since $y_{2}(t)$ is continuous and bounded and not affected by impulses, we conclude that $y_{2}(t)$ is uniformly continuous. Hence there exists a constant $T_{4}$ $\left(0<T_{4}<\tau\right.$, and $T_{4}$ is independent of the choice of $\left.t^{*}\right)$ such that $y_{2}(t)>m_{1} * / 2$ for all $t^{*} \leq t \leq t^{*}+T_{4}$.

If $\theta^{*} \leq T_{4}$, our aim is obtained.

If $T_{4}<\theta^{*} \leq \tau$, from the second equation of (2), we have that

$$
\begin{array}{r}
\frac{d y_{2}(t)}{d t} \geq-\left(\gamma+f_{2} L\right) y_{2}(t), \\
t^{*}<t \leq t^{*}+\theta^{*}
\end{array}
$$

Then we have $y_{2}(t) \geq m_{1}^{*} e^{-\left(\gamma+f_{2} L\right) \tau}$ for $t^{*}<t \leq t^{*}+\theta^{*} \leq t^{*}+\tau$. It is clear that $y_{2}(t) \geq m^{*}$ for $t^{*}<t \leq t^{*}+\theta^{*}$.

If $\theta^{*}>\tau$, by the second equation of (2), then we have that $y_{2}(t) \geq m^{*}$ for $t^{*}<t \leq t^{*}+\tau$. The same arguments can be continued, and we can obtain $y_{2}(t) \geq m^{*}$ for $t^{*}+\tau<t \leq t^{*}+$ $\theta^{*}$. Since the interval $\left[t^{*}, t^{*}+\theta^{*}\right]$ is arbitrarily chosen, we get that $y_{2}(t) \geq m^{*}$ for $t$ large enough. In view of our arguments above, the choice of $m^{*}$ is independent of the positive solution of (2). This completes the proof.

Theorem 10. If $R_{2}>1$, the system (2) is permanent.

Proof. Suppose that $\left(x(t), y_{1}(t), y_{2}(t), c_{0}(t), c_{e}(t)\right)$ is any positive solution of system (2) with initial conditions (2). By (39), we have $x(t) \geq \sigma$ for $t$ large enough. By Theorem 9, we have $y_{2}(t) \geq m^{*}$ for $t$ large enough. From the second equation of system (2), we obtain

$$
\begin{aligned}
\frac{d y_{1}(t)}{d t} \geq & \lambda \frac{q \sigma m^{*}}{1+\alpha \sigma}-\lambda e^{-d \tau} \frac{q \sigma m^{*}}{1+\alpha \sigma} \\
& -d y_{1}(t)-f_{1} y_{1}(t) \\
= & \lambda\left(1-e^{-d \tau}\right) \frac{q \sigma m^{*}}{1+\alpha \sigma} \\
& -\left(d+f_{1}\right) y_{1}(t) \\
\doteq & H-\left(d+f_{1}\right) y_{1}(t) .
\end{aligned}
$$

So,

$$
\begin{aligned}
y_{1}(t) & \geq \frac{H}{d+f_{1}}+\left(y(0)-\frac{H}{d+f_{1}}\right) e^{-\left(d+f_{1}\right) t} \\
& \longrightarrow \frac{H}{d+f_{1}}, \quad \text { as } t \longrightarrow \infty
\end{aligned}
$$

Thus $y_{1}(t) \geq H /\left(d+f_{1}\right)$ for $t$ large enough. By Lemma 2 , we know for a sufficiently small positive $\epsilon_{3}, c_{e}(t)>c_{e}^{*}(t)-\epsilon_{3} \geq$ $\left(b e^{-h T}\right) /\left(1-e^{-h T}\right)-\epsilon_{3}>0$. Then from (15), Lemma 3 , and Definition 8 , we have that system (2) is permanent. The proof is completed.

\section{Discussion}

In this paper, we discuss a pest control model with stage structure for natural enemy in a polluted environment by introducing a constant periodic pollutant input and killing pest at different fixed moments. From Theorems 7, 9, and 10, we can observe that the extinction and permanence of the population are very much dependent on $b, T$, and $\delta$.

To verify the theoretical results obtained in this paper, in the following we will give some numerical simulations and take $\beta=0.9 ; K=0.8 ; q=0.8 ; d=0.4 ; \tau=0.5 ; \lambda=0.8 ; \gamma=$ $0.3 ; b=0.1 ; k=0.5 ; f_{2}=0.2 ; f_{1}=0.1 ; \delta=0.1 ; \alpha=$ $0.2 ; h=2 ; g=0.2 ; m=0.4 ; T=1$ (see Figure 1), and here 

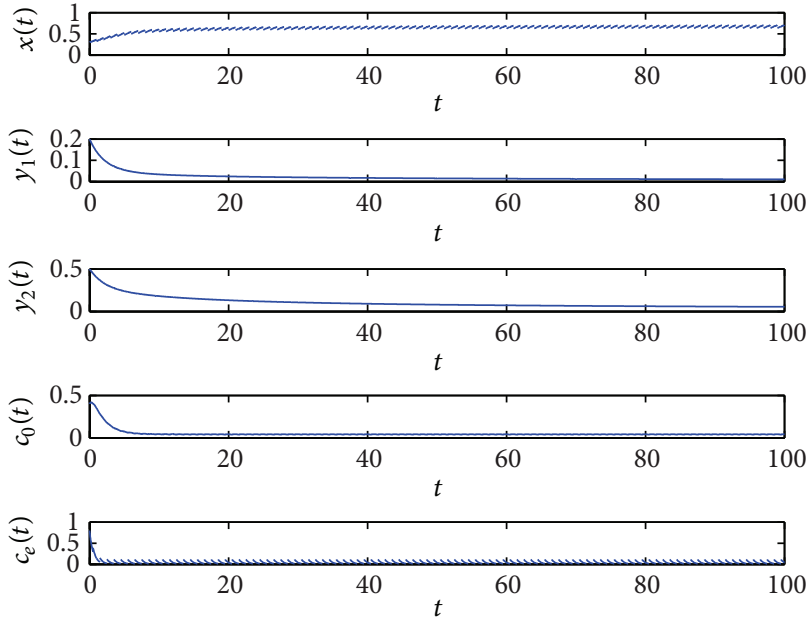

Figure 1: Time series of system (2) with parameters $\beta=0.9, K=$ $0.8, q=0.8, d=0.4, \tau=0.5, \lambda=0.8, \gamma=0.3, b=0.1, k=$ $0.5, f_{2}=0.2, f_{1}=0.1, \delta=0.1, \alpha=0.2, h=2, g=0.2, m=0.4$, and $T=1$.
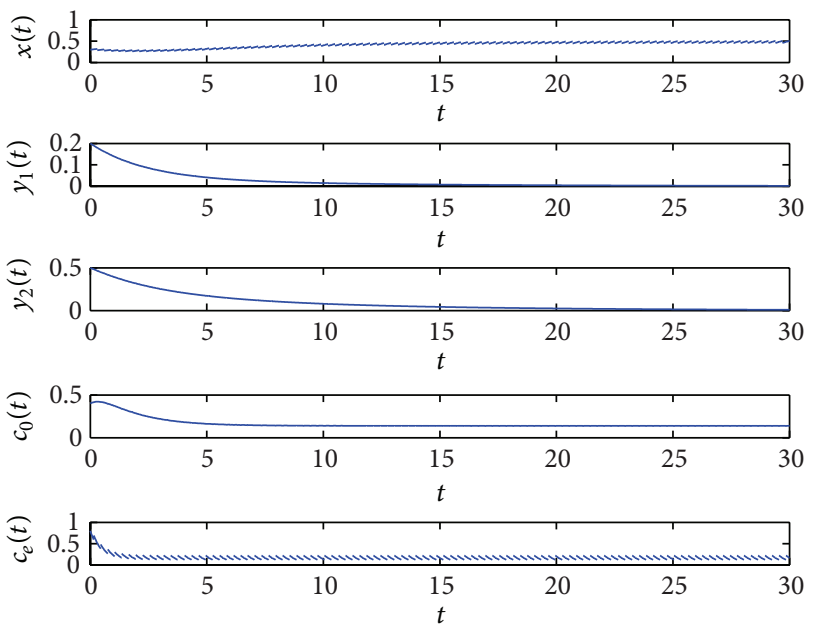

FIgURE 2: Time series of system (2) with parameters $\beta=0.9, K=$ $0.8, q=0.8, d=0.4, \tau=0.5, \lambda=0.8, \gamma=0.3, b=0.1, k=$ $0.5, f_{2}=0.2, f_{1}=0.1, \delta=0.1, \alpha=0.2, h=2, g=0.2, m=0.4$, and $T=0.3$.

we can compute $R_{2}=1.005252>1$, and from Theorem 10 we know the system (2) is permanent. If we decrease the period of pulsing $T=0.3\left(R_{1}=0.755095<1\right)$ or increase the pollution input amount to $b=0.8\left(R_{1}=0.996079<1\right)$, and other parameters are the same with those in Figure 1, the natural enemy will be extinct (see Figures 2 and 3). If we increase the harvesting rate of pests to $\delta=0.5$, and other parameters are the same with those in Figure 1 , then $R_{1}=$ $0.413158<1$, and the natural enemies will also be extinct (see Figure 4). Our results indicate that if impulsive period $T$ is short or $b$ or $\delta$ is too large, the natural enemy will go extinct, but we wish to protect natural enemy from extinction, so we should harvest the pests reasonably and control the period and quantity of emission of pollution into the environment
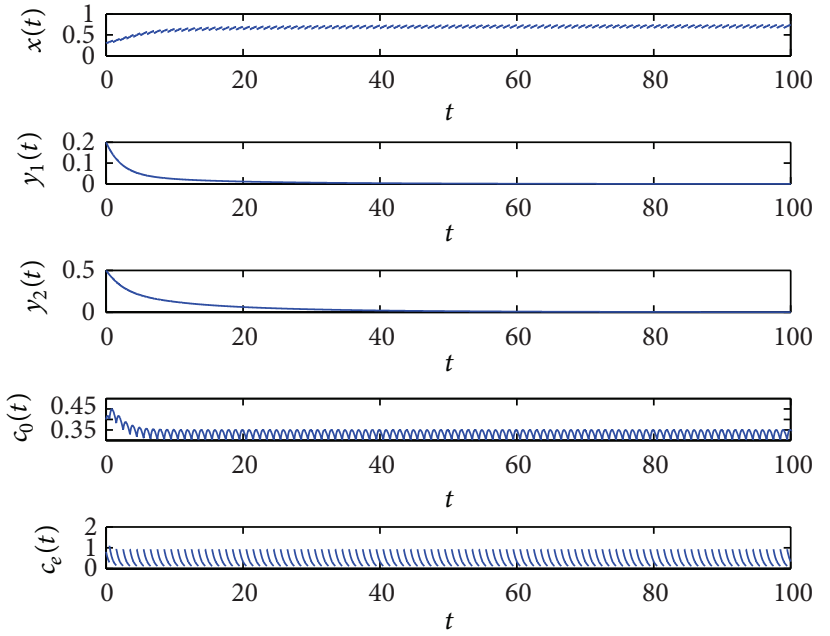

FIgURE 3: Time series of system (2) with parameters $\beta=0.9, K=$ $0.8, q=0.8, d=0.4, \tau=0.5, \lambda=0.8, \gamma=0.3, b=0.8, k=$ $0.5, f_{2}=0.2, f_{1}=0.1, \delta=0.1, \alpha=0.2, h=2, g=0.2, m=$ 0.4 , and $T=1$.
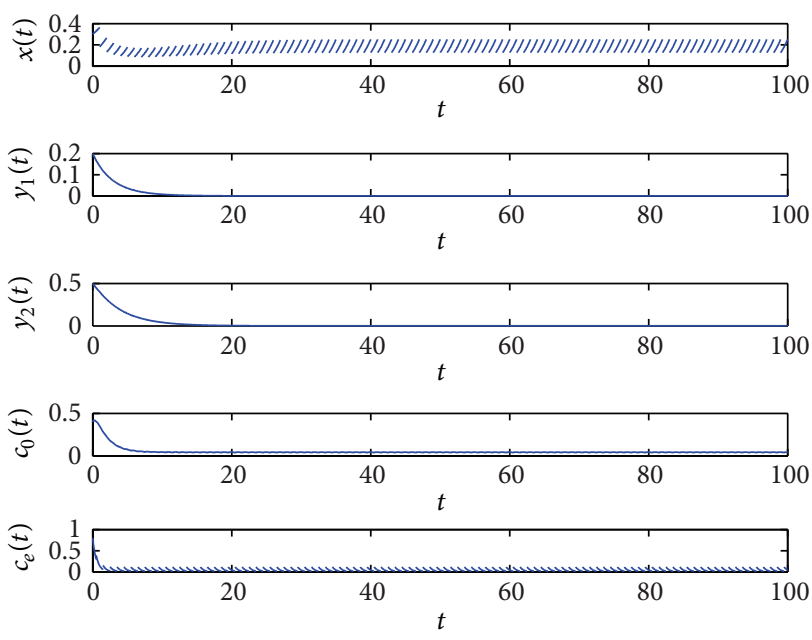

FIgURE 4: Time series of system (2) with parameters $\beta=0.9, K=$ $0.8, q=0.8, d=0.4, \tau=0.5, \lambda=0.8, \gamma=0.3, b=0.1, k=$ $0.5, f_{2}=0.2, f_{1}=0.1, \delta=0.5, \alpha=0.2, h=2, g=0.2, m=$ 0.4 , and $T=1$.

efficiently. This offers us some reasonable suggestions for pest management.

\section{Acknowledgments}

This work is supported by National Natural Science Foundation of China (10971001, 11371030) and the Excellent Talents Support Project of Universities and Colleges in Liaoning.

\section{References}

[1] B. Liu, L. S. Chen, and Y. J. Zhang, "The effects of impulsive toxicant input on a population in a polluted environment," Journal of Biological Systems, vol. 11, no. 3, pp. 265-274, 2003. 
[2] B. Liu, L. Zhang, and Q. Zhang, "The effects of a single stagestructured population model with impulsive toxin input and time delays in a polluted environment," Applicable Analysis, vol. 88, no. 8, pp. 1143-1155, 2009.

[3] M. Liu and K. Wang, "Survival analysis of stochastic singlespecies population models in polluted environments," Ecological Modelling, vol. 220, no. 9-10, pp. 1347-1357, 2009.

[4] Z. Ma, Z. Luo, Z. Jin, and H. Zhao, "The threshold of survival for predator-prey Volterra system of three species in a polluted environment," Systems Science and Mathematical Sciences, vol. 8, no. 4, pp. 373-382, 1995.

[5] M. Zhien, Z. Wengang, and L. Zhixue, "The thresholds of survival for an $n$-dimensional food chain model in a polluted environment," Journal of Mathematical Analysis and Applications, vol. 210, no. 2, pp. 440-458, 1997.

[6] M. Debasis, "Persistence and global stability of a population in a polluted environment with delay," Journal of Biological Systems, vol. 10, no. 3, pp. 225-232, 2002.

[7] A. K. Pal and G. P. Samanta, "A single species population in a polluted environment," International Journal of Biomathematics, vol. 3, no. 2, pp. 187-204, 2010.

[8] F. Tao and B. Liu, "Dynamic behaviors of a single-species population model with birth pulses in a polluted environment," The Rocky Mountain Journal of Mathematics, vol. 38, no. 5, pp. 1663-1684, 2008.

[9] Y. Xiao and L. Chen, "How do the spatial structure and time delay affect the persistence of a polluted species," Applicable Analysis, vol. 82, no. 3, pp. 253-267, 2003.

[10] X. Yang, Z. Jin, and Y. Xue, "Weak average persistence and extinction of a predator-prey system in a polluted environment with impulsive toxicant input," Chaos, Solitons \& Fractals, vol. 31, no. 3, pp. 726-735, 2007.

[11] Z. Zhao, L. Chen, and X. Song, "Extinction and permanence of chemostat model with pulsed input in a polluted environment," Communications in Nonlinear Science and Numerical Simulation, vol. 14, no. 4, pp. 1737-1745, 2009.

[12] A. Hastings, "Age-dependent predation is not a simple process. I. Continuous time models," Theoretical Population Biology, vol. 23, no. 3, pp. 347-362, 1983.

[13] A. Hastings, "Delays in recruitment at different trophic levels: effects on stability," Journal of Mathematical Biology, vol. 21, no. 1, pp. 35-44, 1984.

[14] W. Wang and L. Chen, "A predator-prey system with stagestructure for predator," Computers \& Mathematics with Applications, vol. 33, no. 8, pp. 83-91, 1997.

[15] S. Gao, L. Chen, and Z. Teng, "Hopf bifurcation and global stability for a delayed predator-prey system with stage structure for predator," Applied Mathematics and Computation, vol. 202, no. 2, pp. 721-729, 2008.

[16] S. A. Gourley and Y. Kuang, "A stage structured predator-prey model and its dependence on maturation delay and death rate," Journal of Mathematical Biology, vol. 49, no. 2, pp. 188-200, 2004.

[17] H. Zhang and L. Chen, "A model for two species with stage structure and feedback control," International Journal of Biomathematics, vol. 1, no. 3, pp. 267-286, 2008.

[18] M. De la Sen, R. P. Agarwal, A. Ibeas, and S. Alonso-Quesada, "On a generalized time-varying SEIR epidemic model with mixed point and distributed time-varying delays and combined regular and impulsive vaccination controls," Advances in Difference Equations, vol. 2010, Article ID 281612, 42 pages, 2010.
[19] M. de la Sen, "On some structures of stabilizing control laws for linear and time-invariant systems with bounded point delays and unmeasurable states," International Journal of Control, vol. 59, no. 2, pp. 529-541, 1994.

[20] X. Song, M. Hao, and X. Meng, "A stage-structured predatorprey model with disturbing pulse and time delays," Applied Mathematical Modelling, vol. 33, no. 1, pp. 211-223, 2009.

[21] X. Meng and L. Chen, "Permanence and global stability in an impulsive Lotka-Volterra $n$-species competitive system with both discrete delays and continuous delays," International Journal of Biomathematics, vol. 1, no. 2, pp. 179-196, 2008.

[22] X. Meng, L. Chen, and H. Cheng, "Two profitless delays for the SEIRS epidemic disease model with nonlinear incidence and pulse vaccination," Applied Mathematics and Computation, vol. 186, no. 1, pp. 516-529, 2007.

[23] B. Liu, Q. Zhang, and Y. Gao, "The dynamics of pest control pollution model with age structure and time delay," Applied Mathematics and Computation, vol. 216, no. 10, pp. 2814-2823, 2010.

[24] Z. Liu and R. Tan, "Impulsive harvesting and stocking in a Monod-Haldane functional response predator-prey system," Chaos, Solitons and Fractals, vol. 34, no. 2, pp. 454-464, 2007.

[25] J. Hui and D. Zhu, "Dynamic complexities for prey-dependent consumption integrated pest management models with impulsive effects," Chaos, Solitons and Fractals, vol. 29, no. 1, pp. 233251, 2006.

[26] Z. Jin, M. Haque, and Q. Liu, "Pulse vaccination in the periodic infection rate SIR epidemic model," International Journal of Biomathematics, vol. 1, no. 4, pp. 409-432, 2008.

[27] B. Liu, Z. D. Teng, and L. S. Chen, "The effect of impulsive spraying pesticide on stagestructured population models with birth pulse," Journal of Biological Systems, vol. 13, no. 1, pp. 3144, 2006.

[28] B. Liu, Y. Zhang, and L. Chen, "Dynamic complexities in a Lotka-Volterra predator-prey model concerning impulsive control strategy," International Journal of Bifurcation and Chaos in Applied Sciences and Engineering, vol. 15, no. 2, pp. 517-531, 2005.

[29] X. Meng and L. Chen, "The dynamics of a new SIR epidemic model concerning pulse vaccination strategy," Applied Mathematics and Computation, vol. 197, no. 2, pp. 582-597, 2008.

[30] S. Tang and L. Chen, "Density-dependent birth rate, birth pulses and their population dynamic consequences," Journal of Mathematical Biology, vol. 44, no. 2, pp. 185-199, 2002.

[31] H. Zhang, P. Georgescu, and L. Chen, "An impulsive predatorprey system with Beddington-DeAngelis functional response and time delay," International Journal of Biomathematics, vol. 1, no. 1, pp. 1-17, 2008.

[32] Y. Kuang, Delay Differential Equations with Applications in Population Dynamics, vol. 191 of Mathematics in Science and Engineering, Academic Press, San Diego, Calif, USA, 1993. 


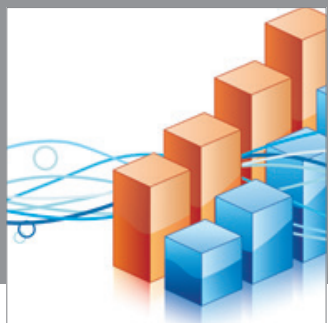

Advances in

Operations Research

mansans

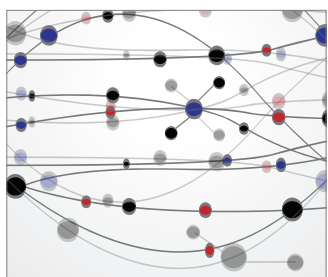

The Scientific World Journal
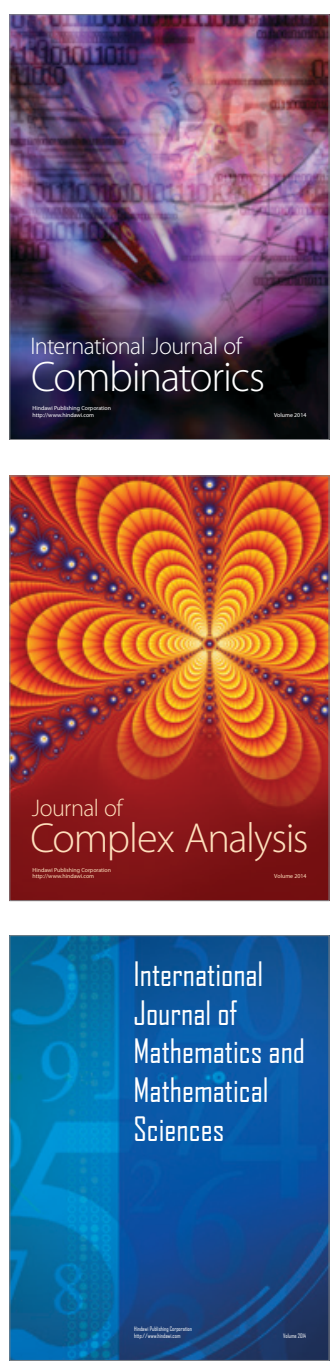
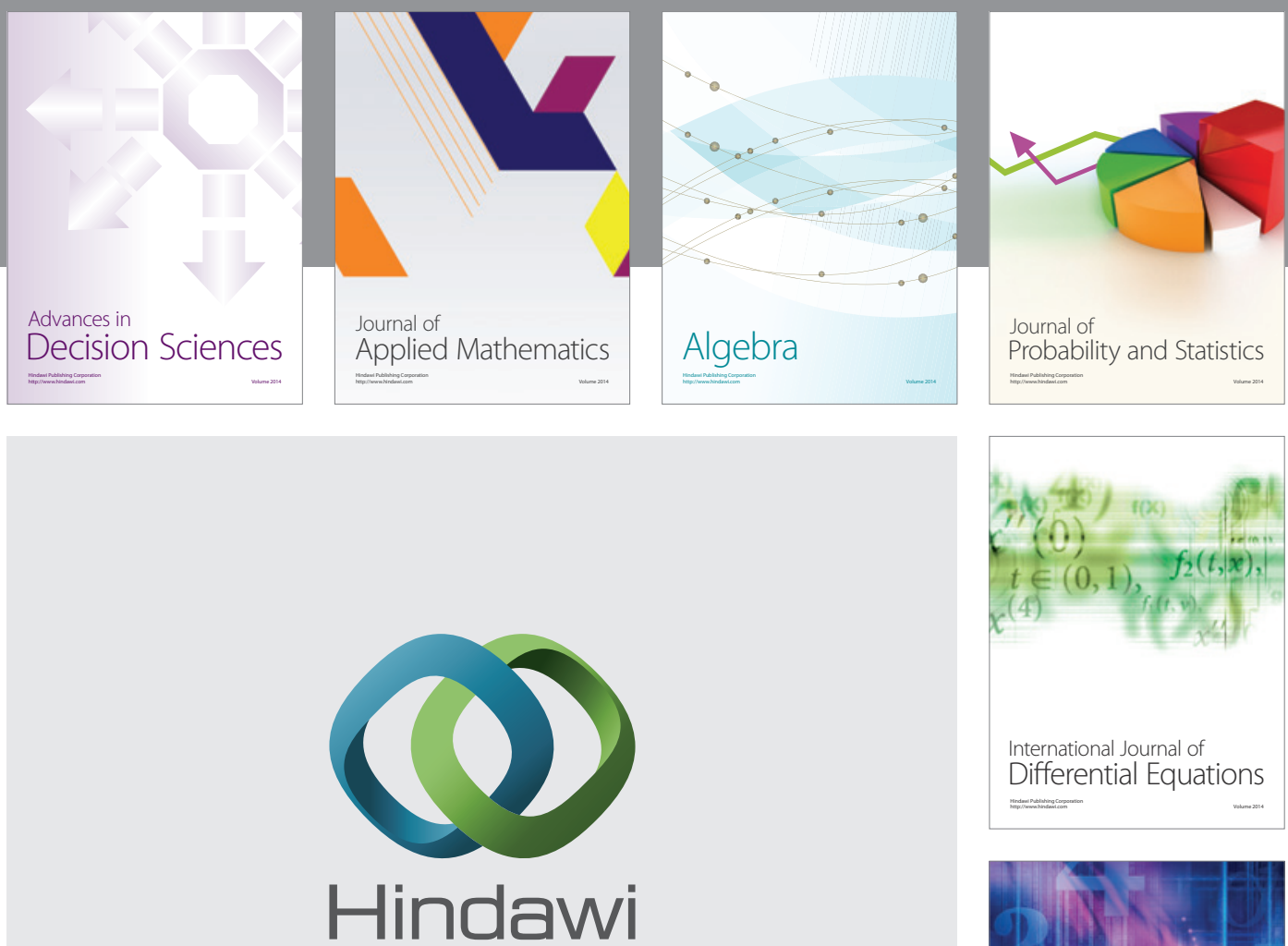

Submit your manuscripts at http://www.hindawi.com
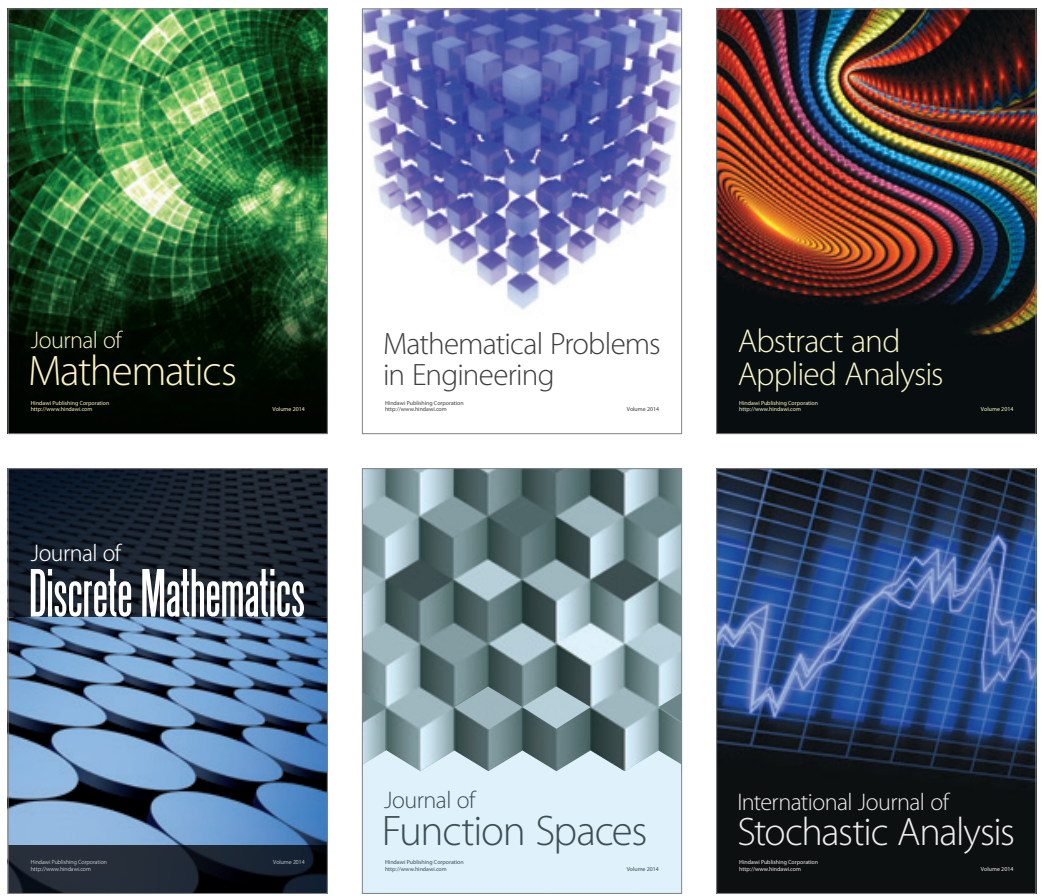

Journal of

Function Spaces

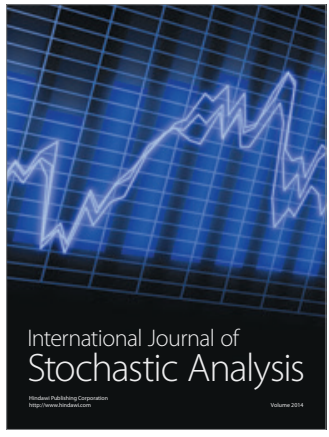

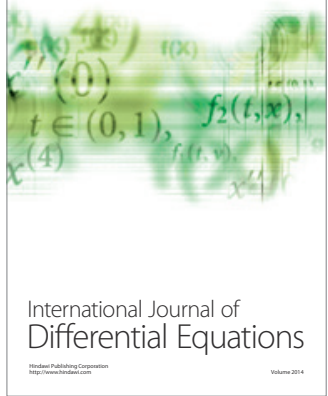
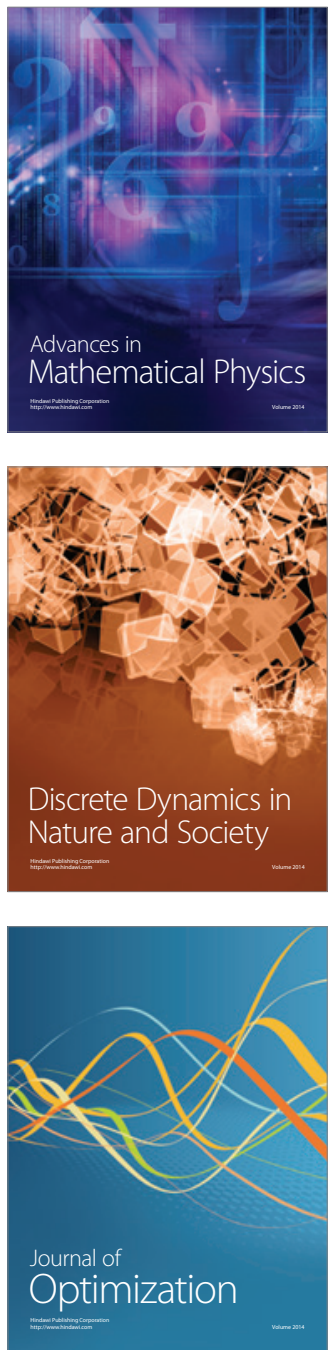